\title{
Airway Y-stent Insertion in Tracheobronchomalacia: A New Experience
}

\author{
Kuan YC, ${ }^{a}$ How SH, ${ }^{a} \mathrm{Ng} \mathrm{TH},{ }^{a}$ Liam CK, ${ }^{\mathrm{b}}$ Mohd Ashri A, ${ }^{\mathrm{c}}$ Mohammed Fauzi AR ${ }^{\mathrm{a}}$ \\ ${ }^{a}$ Department of Internal Medicine, International Islamic University Malaysia, Kuantan. \\ ${ }^{b}$ Department of Medicine, University Malaya Medical Centre, Kuala Lumpur. \\ 'Department of Anaesthesiology, International Islamic University Malaysia, Kuantan.
}

\section{ABSTRACT:}

This case report describes a rigid bronchoscopy-assisted placement of a silicone airway Y-stent in a patient who developed tracheobronchomalacia following repeated dilatations for post-tuberculous airway stenosis. This is the first report of an airway Y-stent insertion in Malaysia.

KEYWORDS: Tracheobronchomalacia, airway stent, tuberculosis.

\section{INTRODUCTION}

Tracheomalacia (TM) is a condition characterized by weakness of the trachea due to impaired supporting structures, i.e. the longitudinal elastic fibres of the pars membranacea and cartilaginous tissue, leading to a softer and more collapsible airway. ${ }^{1}$ If the bronchus is also involved, the term tracheobronchomalacia (TBM) is used. We describe a case of a lady, who had endobronchial tuberculosis complicated by tracheobronchial stenosis. She underwent repeated bronchoscopy-assisted dilatations using Controlled Radial Expansion (CRE) (Boston Scientific, Massachusetts, USA) balloon. Following repeated dilatations, she developed tracheobronchomalacia, which required rigid bronchoscopy-assisted insertion of an airway Y-stent.

\section{CASE REPORT}

The patient was a 38-year-old lady who presented with chronic cough and stridor. Her sputum acid-fast bacilli examination was negative. The initial flexible bronchoscopy found a lower tracheal stenosis of $4 \mathrm{~cm}$ in length, a stenosed and swollen right main bronchus, and a pin-hole stenosis of the right bronchus intermedia. She was diagnosed with endobronchial tuberculosis based on clinical and radiographic features. She received oral prednisolone for one month and anti-tuberculous therapy for a total of nine-month duration.

However, she was still stridorous and had reduced effort tolerance even after completion of treatment. A repeat bronchoscopy showed mild-to-moderate

Corresponding author:

Dr Kuan Yeh Chunn

Department of Internal Medicine,

International Islamic University Malaysia,

Jalan Hospital, 25100 Kuantan, Pahang, Malaysia.

Tel: 609-5132797

Fax: 609-5171897

E-mail: kychunn@yahoo.com stenosis of the lower trachea measuring $2.5 \mathrm{~cm}$ in length and stenosis of the right bronchus intermedia, which was dilated using the Controlled Radial Expansion (CRE) balloon to $6 \mathrm{~mm}$. Subsequently, she had seven sessions of CRE dilatations performed on the right bronchus intermedia, which was dilated to the maximum of $12 \mathrm{~mm}$. She also had two CRE dilatations of the lower trachea to the maximum of $10 \mathrm{~mm}$. Her symptoms improved after each dilatation procedure but always recurred a few months later. Just prior to the last CRE dilatation, her spirometry showed $\mathrm{FEV}_{1} 0.38 \mathrm{~L}$ (14\% of predicted), FVC $2.10 \mathrm{~L}$ (65\% of predicted) and $\mathrm{FEV}_{1} / \mathrm{FVC}$ ratio of $18.1 \%$. She could walk $180 \mathrm{~m}$ on the 6-minute walk test (6MWT) without dropping her oxygen saturation (pre-6MWT SpO2 98\% and heart rate 79 beats/minute, post-6MWT SpO2 99\% and heart rate 94 beats/minute).

In view of recurrent symptoms and airway stenosis, she was referred for airway stenting. Under general anaesthesia, a rigid bronchoscopic examination showed mild stenoses of the lower trachea (measuring $5.5 \mathrm{~cm}$ in length and $10 \mathrm{~mm}$ in diameter) and right bronchus intermedia. She also had severe lower tracheomalacia and right main bronchomalacia: The tracheal and bronchial endoluminal space was narrowed because opposing walls were partially collapsed causing partial occlusion of the airway (Figure 1).

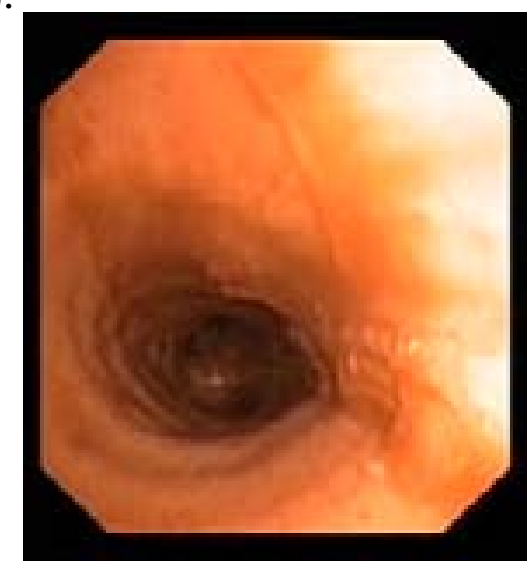

Figure 1: Tracheomalacia causing narrowing of tracheal lumen 
Serial rigid endoscopic dilatations of the lower trachea were performed using rigid bronchoscope size $10 \mathrm{~mm}, 12 \mathrm{~mm}$ and $13.2 \mathrm{~mm}$. Mild laceration and minimal bleeding were noted in the proximal end of the tracheal stenosis. A silicone airway Y-stent (Novatech, Boston Medical, Massachusetts, USA) size 15-12-12 mm was inserted and both bronchial sleeves were deployed satisfactorily (Figure 2). Right upper lobe bronchus was mildly oedematous due to stent manipulation. She was extubated immediately after the procedure and transferred to the ward for postprocedural recovery and observation.

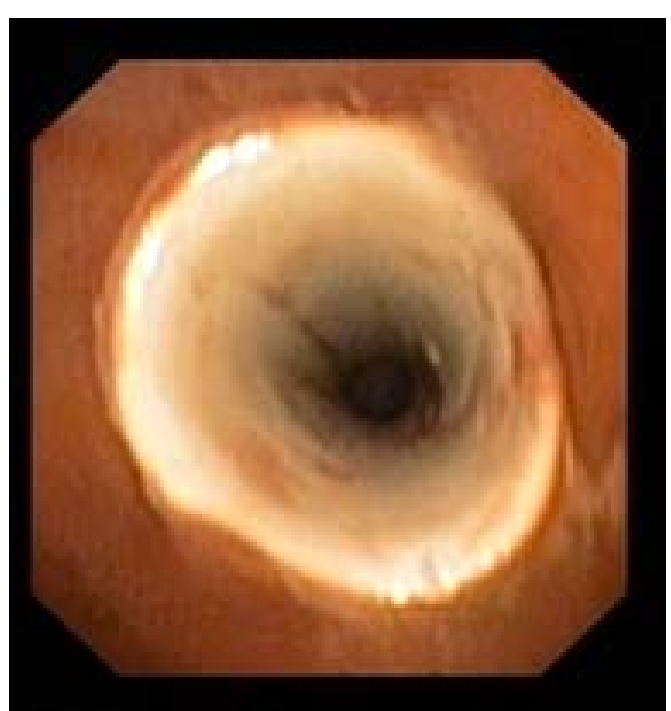

Figure 2: Airway Y-stent stent inserted, proximal view

A post-procedure chest $\mathrm{X}$-ray revealed mild pneumomediastinum, which resolved spontaneously on the subsequent chest radiograph a week later. She was given I.V. dexamethasone $8 \mathrm{mg}$ three times a day for the first three days and continued oral dexamethasone $8 \mathrm{mg}$ three times a day for a total duration of one week. She also received I.V. ceftriaxone $1 \mathrm{~g}$ daily for one week. Spirometry performed a week after the procedure showed marked improvement: $\mathrm{FEV}_{1} 1.99 \mathrm{~L}$ (69\% of predicted), FVC $2.51 \mathrm{~L}$ (73\% of predicted) and $\mathrm{FEV}_{1} / \mathrm{FVC}$ ratio of $79 \%$. She was no longer stridorous and her functional status improved. She could attend to her usual daily activities, which she required assistance from a caregiver previously.

She was discharged well and planned for reassessment of her airway after six months. In the interim, she frequently complained of excessive secretions, which were alleviated using regular nebulized saline. Check bronchoscopy performed every one to two months also showed moderate build-up of granulation tissue at the lower end of the right limb of the airway Y-stent.

Six months later, she underwent repeat rigid bronchoscopy for reassessment of her airway. The airway Y-stent was seen in-situ. After removal of the airway $Y$-stent, significant lower tracheomalacia and right main bronchomalacia were still present. volume 11 Number 1 June 2012
There was a moderate amount of granulation tissue in the right main bronchus and only minimal amount seen in the left main bronchus. Photocoagulation and grasp forceps were used to remove the excessive granulation tissue build-up in the right main bronchus. A new airway Y-stent (Novatech, Boston Medical, Massachusetts, USA) size 16-13-13 (cut to 5.8 $\mathrm{cm}$ (trachea) $-0.6 \mathrm{~cm}$ (left limb) $-1.0 \mathrm{~cm}$ (right limb) in lengths) was inserted. The patient recovered from the procedure and was discharged well.

She was to be followed up with bronchoscopy every one-to-two months and airway stent replacement every six-to-twelve months.

\section{DISCUSSION}

TM and TBM in adults can be classified into primary (congenital) and secondary (acquired) forms. ${ }^{1,2}$ Congenital causes include polychondritis, idiopathic "giant trachea" or Mounier-Kuhn Syndrome while acquired causes include post-traumatic, e.g. postintubation, post-traumatic and post-lung transplantation, chronic infection, chronic inflammation and chronic external compression of the trachea from aneurysms, tumours, cysts and abscesses. ${ }^{1}$

Although endoluminal tuberculosis leads to airway stenosis, it can also cause weakening and destruction of the airway and supporting cartilages. According to Feist et al., ${ }^{2}$ the most common cause of acquired malacia of the airway is tracheostomy or intubation with endotracheal tubes. Possible mechanisms that were implicated in causing tracheal weakness include pressure necrosis, disruption of blood supply, friction on, and inflammation of, the mucosa. ${ }^{1}$

In this case, the patient's tracheal and bronchial walls were weakened from the tuberculous disease process. With further repeated attempts at tracheobronchial dilatation, the stenosed segments were temporarily relieved but at the expense of worsening malacia of the affected tracheobronchial segments. This wasseen on rigidbronchoscopicexamination, which revealed only mild stenotic disease but severe lower TM and right main bronchomalacia. Severity of TM can be classified into mild, moderate or severe depending on the degree of tracheal obstruction during expiration. ${ }^{3}$ The TM is considered mild if the obstruction is one-half of the lumen, moderate if it is three-quarters and severe if the anterior and posterior walls touched.

Airway stents can be inserted to keep the malacic airway's patent. Silicone stents are preferred to metallic ones because the latter are subjected to excessive granulation tissue formation and material fragmentation with time, which can cause airway obstruction, perforation and death. ${ }^{1}$

Furthermore, metallic stents are harder to remove and make future surgical interventions difficult. 
The patient had a silicone Y-stent placed in the lower trachea and the sleeves deployed into the main bronchi. Our PubMed database search had shown no previous report of an airway $Y$-stent insertion under rigid bronchoscopy in Malaysia. Pang et al. described ten cases of self-expandable metallic stents inserted through flexible bronchoscopy but they were all straight airway stents. ${ }^{4}$

The patient's symptoms and spirometry results showed significant improvement immediately following the stent placement. She will require long-term follow-up with bronchoscopy and review of her symptoms and lung function. One study reported that at mean 15 months after stent placement for stenotic and malacic lesions, peak flow parameters had declined though patients' subjective improvement continued. ${ }^{5}$ In suitable candidates, surgery i.e. tracheoplasty, may also be considered as a definitive treatment option besides long-term stenting. ${ }^{1}$

\section{REFERENCES}

1. Carden KA, Boiselle PM, Waltz DA, Ernst A. Tracheomalacia and tracheobronchomalacia in children and adults: an in-depth review. Chest 2005;127:984-1005.

2. Feist JH, Johnson TH, Wilson RJ. Acquired tracheomalacia: etiology and differential diagnosis. Chest 1975; 68:340-5.

3. Nuutinen J. Acquired tracheobronchomalacia: a clinical study with bronchological correlations. Ann Clin Res 1977;9:350-5.

4. Pang YK, Liam CK, Leow CH, et al. Tracheobronchial stenting is safe and effective in relieving upper airway obstruction. Med J Malaysia 2006;61:147-50.

5. Gotway MB, Golden JA, LaBerge JM, et al. Benign tracheobronchial stenoses: changes in short-term and long-term pulmonary function testing after expandable metallic stent placement. J Comput Assist Tomogr 2002;26:564-72. 
THE INTERNATIONAL MEDICAL JOURNAL MALAYSIA

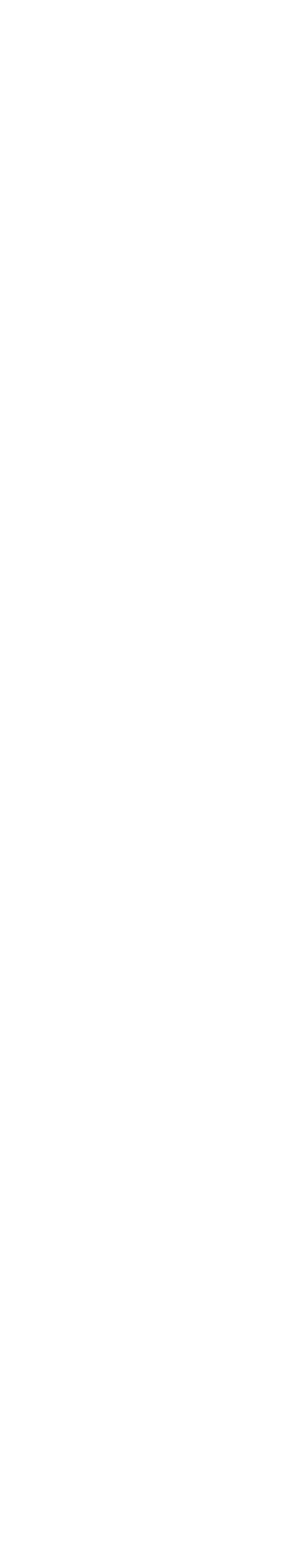
(1) 